\title{
X-Band/C-Band-Comparison of Ship Wake Detectability
}

\author{
Björn Tings ${ }^{\mathrm{a}}$, Sven Jacobsen ${ }^{\mathrm{a}}$, Stefan Wiehle ${ }^{\mathrm{a}}$, Egbert Schwarz ${ }^{\mathrm{b}}$, Holger Daedelow $^{\mathrm{b}}$

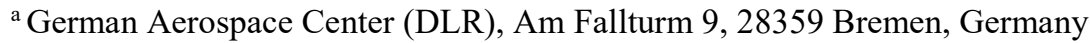 \\ ${ }^{\mathrm{b}}$ German Aerospace Center (DLR), Kalkhorstweg 53, 17235 Neustrelitz, Germany
}

\begin{abstract}
Recent studies investigated the detectability of ship wake signatures on SAR imagery using a large number of SAR images collocated with Automatic Identification System data for training machine learning models. These detectability models are in agreement with oceanographic expectations from preceding studies and can therefore be used for comparing the performance of different SAR sensors in terms of wake detectability. Previous model comparisons showed better wake detection performance of TerraSAR-X (TS-X) than of RADARSAT-2 (RS2) and Sentinel-1 (S1). A comparison between CosmoSkymed (CSK) and RS2 is performed here, to examine the hypothesis that X-Band is generally better for wake detection than C-Band. Finally, this hypothesis is not confirmed, as the detectability models for TS-X, CSK and RS2 reveal similar performances. A comparison of wake detection performance should take the individual wake components into account separately.
\end{abstract}

\section{Introduction}

Modern SAR-based ship detection algorithms also exploit the visibility of ship wake signatures generated by moving vessels in order to increase the information content [1]. The SAR-imaging of ship generated sea surface waves, i.e. ship wakes, has been explained by researchers through theoretical considerations of the underlying physics on the basis of qualitative image analysis as well as simulation models, e.g. in [2]. The detectability of such ship wake signatures is dependent on various influencing parameters, which can be categorized into environmental conditions, ship properties or image acquisition settings [2]. Recent studies show that the dependency of wake detectability on the influencing parameters can be modelled by machine learning (ML) methods and that the resulting models are mainly in agreement with the oceanographic expectations defined by researchers through simulation or theoretical considerations $[3,4,5]$.

In [3] was described that wakes are better detectable using the TerraSAR-X (TS-X) SAR sensor in comparison to the Sentinel-1 (S1) or RADARSAT-2 (RS2) SAR sensors. However, no explanation for this outcome could be given in [3], as TS-X differs by two main properties compared to S1 and RS2: the orbit altitude (TS-X operates around $200 \mathrm{~km}$ lower) and the radar frequency (TS-X uses X-Band SAR, while S1 and RS2 use C-Band SARs). In this study the missing explanation is investigated by including the CosmoSkyMed (CSK) SAR sensor, which operates on a similar orbit altitude than S1 and RS2, but uses an X-Band SAR.

The next section summarizes the updated results from [3] and [4] and presents the new results obtained with CSK. In section 3 a qualitative comparison of RS2 and CSK is presented. The results of this study are discussed in section 4 and concluded at the end of the paper in section 5 .

\section{ML-based detectability models}

The models presented in [3] and [4] are updated using a training dataset with an increased size of around 2700 TS-X wake samples having passed additional manual inspection. Wake candidates showing either of the following wake components are considered as detected: turbulent wake, Kelvin wake, V-narrow wake, or ship-generated internal wave. This means, wake candidates consisting only of near-hull turbulences or showing neither of the above wake components are considered as not detected. Detailed information about the identification of wake component and extraction of the influencing parameters can be found in [5].

In detail, the amounts wake samples in the respective datasets are specified in Table 1:

\begin{tabular}{|l|l|}
\hline SAR sensor & Amount of wake samples \\
\hline TS-X & 2684 (HH: 2267; VV: 417) \\
\hline RS2 & 387 (HH-polarization) \\
\hline S1 & 556 (VV-polarization) \\
\hline CSK & 82 (HH-polarization) \\
\hline
\end{tabular}

Table 1 Amount of wake samples for each SAR sensor in the respective datasets

In the following subsections the creation of the models is briefly described. More detailed information can be found in the respective publications [3], [4] and [5].

\subsection{Linear wake detectability models}

The amount of RS2, S1 and CSK data available for this study was only sufficient for the training of a linear wake detectability model. Therefore, the comparison of wake detectability between the sensors can only be based on this simplified approach, as it was already done in [3]. This 
means, only the influencing parameters, which showed a linear (i.e. independent monotonic) influence on the wake detectability in [4] and [5], are considered for comparison. Briefly, the linear detectability models are created using the following steps:

1. Identifying locations of moving vessels in SAR images using collocated Automatic Identification System (AIS) data

2. Extraction of training dataset of wake samples at identified locations

3. Labelling with class labels if one relevant wake components have been "detected" or "not detected"

4. Extraction of influencing parameters from SAR image and AIS:
1. Incidence-Angle,
2. AIS-Vessel-Velocity,
3. AIS-Length and
4. SAR-Wind-Speed

5. Training of linear Support Vector Machine (SVM) classifiers for discrimination between the two classes using all four influencing parameters as input features

6. Probability of class affiliation to class "detected" calculated by a classifier for a sample setting of influencing parameters defines the probability of detection i.e. quantifies the detectability for the respective parameter set

Finally, 2D-detectablity charts are created by binning and then sampling the whole feature space of the influencing parameters. The classification model is then used to calculate the probability of detection for each sample setting of influencing parameters. The probabilities are then color-coded and the colors plotted into 2-dimensional coordinate systems to generate a view into the model according to the underlying influencing parameters. Inspecting the classification models by these views into the models gives information about the characteristics of influence of the respective influencing parameters on detectability. Also, the overall wake detection performance of the respective sensors can be assessed. A selection of 2D-detectabilty charts for comparing TS-X, S1 and RS2 is presented in the following Figure 1 to Figure 4.

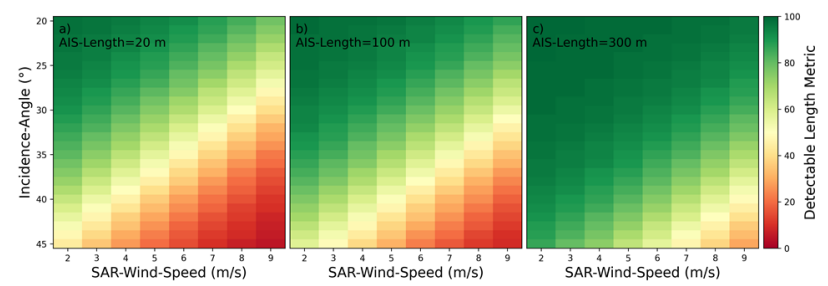

Figure 1 TS-X (HH) high-resolution 2D-detectability charts based on SAR-Wind-Speed, Incidence-Angle and from left to right $20 \mathrm{~m}, 100 \mathrm{~m}$, and $300 \mathrm{~m}$ AIS-Length.

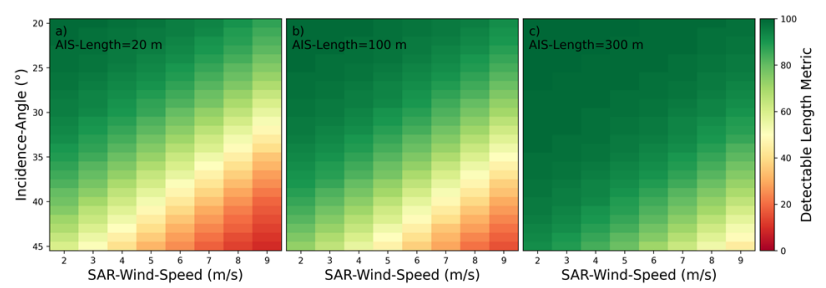

Figure 2 TS-X (VV) high-resolution 2D-detectability charts based on SAR-Wind-Speed, Incidence-Angle and from left to right $20 \mathrm{~m}, 100 \mathrm{~m}$, and $300 \mathrm{~m}$ AIS-Length.

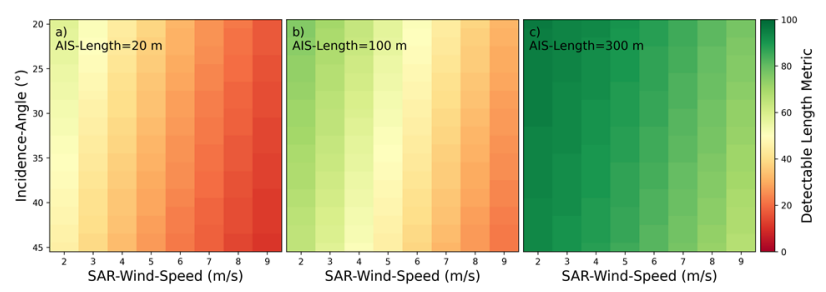

Figure 3 S1 (VV) medium-resolution 2D-detectability charts based on SAR-Wind-Speed, Incidence-Angle and from left to right $20 \mathrm{~m}, 100 \mathrm{~m}$, and $300 \mathrm{~m}$ AIS-Length

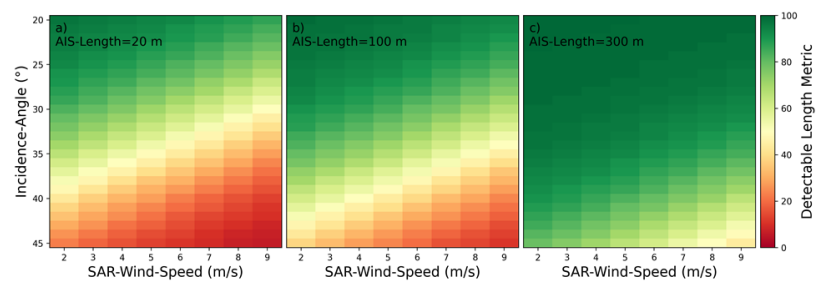

Figure 4 RS2 (HH) high-resolution 2D-detectability charts based on SAR-Wind-Speed, Incidence-Angle and from left to right $20 \mathrm{~m}, 100 \mathrm{~m}$, and $300 \mathrm{~m}$ AIS-Length

The results from a statistical model for wake detectability using CSK data are presented for the first time in this study. Two views with different influencing parameters are shown in the Figure 5 and Figure 6.

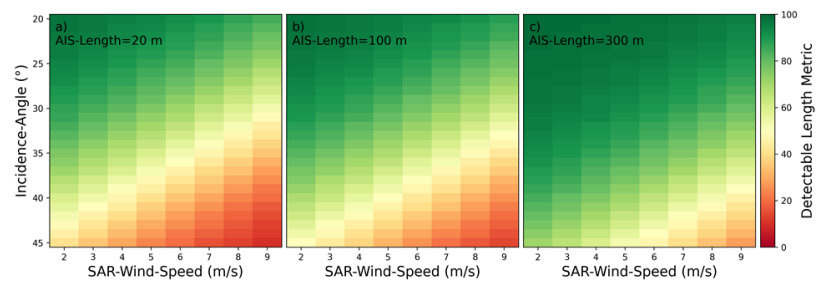

Figure 5 CSK (HH) high-resolution 2D-detectability charts based on SAR-Wind-Speed, Incidence-Angle and from left to right $20 \mathrm{~m}, 100 \mathrm{~m}$, and $300 \mathrm{~m}$ AIS-Length

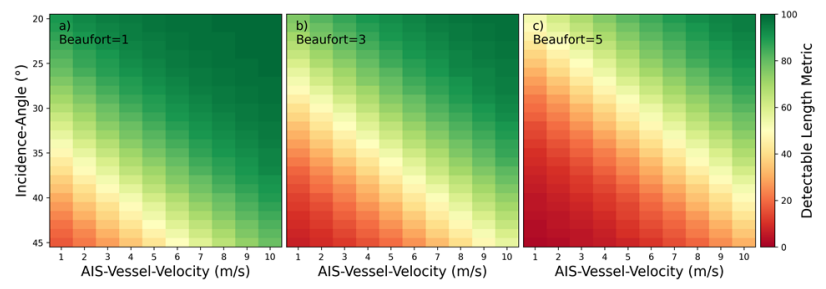

Figure 6 CSK (HH) high-resolution 2D-detectability charts based on AIS-Vessel-Velocity, Incidence-Angle and from left to right $1 \mathrm{bft}, 3 \mathrm{bft}$ and $5 \mathrm{bft}$ Beaufort numbers 
The characteristics of influences of the influencing parameters Incidence-Angle, AIS-Vessel-Velocity, AIS-Length and SAR-Wind-Speed on the detectability of wakes are in agreement for the four different sensors. For AIS-Vessel-Velocity as well as AIS-Length positive independent monotonic influence is reproduced by the models and for Incidence-Angle as well as SAR-Wind-Speed negative independent monotonic influence. The overall detectability is lower for $\mathrm{S} 1$ in comparison to RS2, TS-X and CSK. Thus, only the assumption from [3] that TS-X is better for detecting wakes than $\mathrm{S} 1$ can be confirmed. It cannot be confirmed that TS-X has superior performance to RS2 in detecting wakes. The detectability model for the CSK sensor does not reproduce significantly better or worse wake detection performance in comparison to RS2 or TS-X.

\subsection{Nonlinear wake detectability models}

The amount of TS-X data available for this study was also sufficient for the training of a nonlinear wake detectability model. This means also influencing parameters with expected nonlinear influence on wake detectability can be modelled. Again, all considered influencing parameter are combined in one model so that interdependencies between all parameters can be investigated.

Some of the model creation steps listed in the previous subection 2.1 are changed as follows:

4. Extraction of additional influencing parameters, which results in a total of nine parameters:
5. AIS-CoG,
6. SAR-Significant-Wave-Height,
7. SAR-Wave-Length,
8. AIS-CoG-SAR-Wave-Direction,
9. AIS-CoG-WRF-Wind-Direction

5. Training of one SVM classifier using a polynomial kernel of degree two

The procedure for generation of 2D-detectablity charts is identical to Section 2.1, except that the sampling of the 9D feature space is more complex and therefore more charts are obtained. A selection of 2D-detectablity charts only based on TS-X high-resolution HH-polarization data are presented in the following Figure 7 to Figure 10.

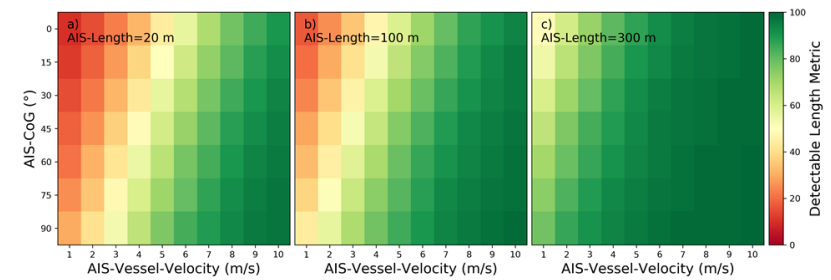

Figure 7 2D-detectability charts based on AIS-Vessel-Velocity, AIS-CoG and from left to right $20 \mathrm{~m}, 100 \mathrm{~m}$ and $200 \mathrm{~m}$ AIS-Length

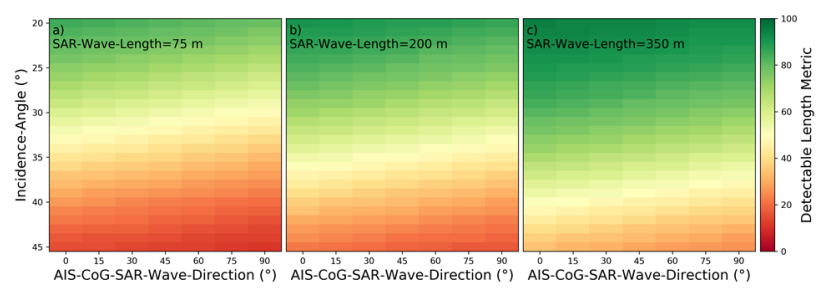

Figure 8 2D-detectability charts based on AIS-CoG-SAR-Wave-direction, Incidence-Angle and from left to right $75 \mathrm{~m}, 150 \mathrm{~m}$ and $300 \mathrm{~m}$ SAR-Wave-Length
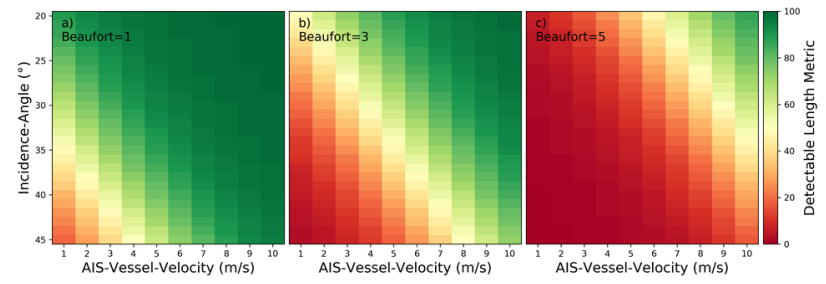

Figure 9 2D-detectability charts based on Incidence-Angle, AIS-Vessel-Velocity and from left to right $1 \mathrm{bft}, 3 \mathrm{bft}$ and $5 \mathrm{bft}$ Beaufort numbers.
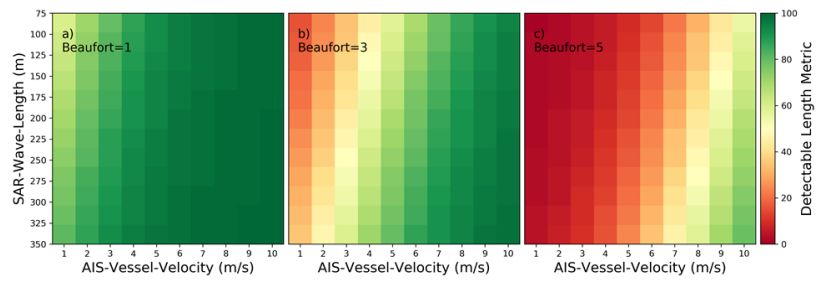

Figure 10 2D-detectability charts based on SAR-Wave-Length, AIS-Vessel-Velocity and from left to right $1 \mathrm{bft}, 3 \mathrm{bft}$ and $5 \mathrm{bft}$ Beaufort numbers.

The characteristics of influences of the influencing parameters Incidence-Angle, AIS-Vessel-Velocity, AIS-Length and SAR-Wind-Speed on the detectability of wakes are for this nonlinear model in agreement with the characteristics reproduced by the linear models in subsection 2.1 .

\section{Qualitative comparison of CSK and RS2}

Examples of CSK acquisitions collocated in space and time with RS2 acquisitions are displayed in the Figure 11 to Figure 13. All six images have similar resolutions and are scaled to an identical value range of the pixel's calibrated amplitudes. All imaged ships travel with velocities considered as high. 


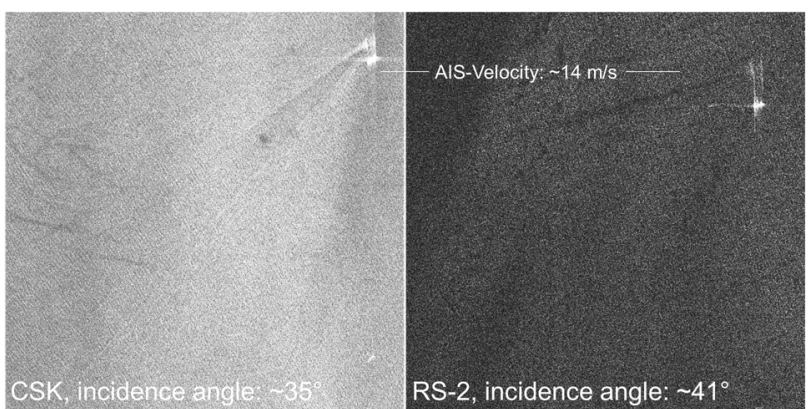

Figure 11 Comparison of CSK and RS-2 images acquired on 25.03.2019 at the same area with $\mathrm{HH}$ polarization and showing the same ship, left: CSK HIMAGE from 17:05 UTC, right: RS-2 Fine from $\sim 16: 47$ UTC

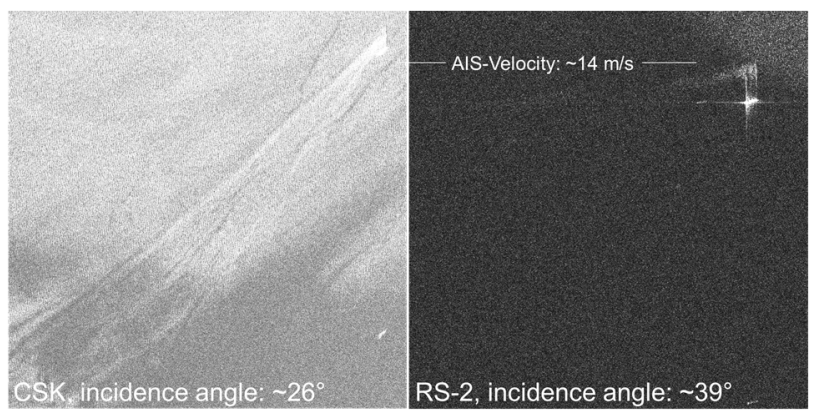

Figure 12 Comparison of CSK and RS-2 images acquired on 25.04.2019 at the same area with $\mathrm{HH}$ polarization and showing the same ship, left: CSK HIMAGE from $\sim 17: 11$ UTC, right: RS-2 Fine from $\sim 16: 43$ UTC

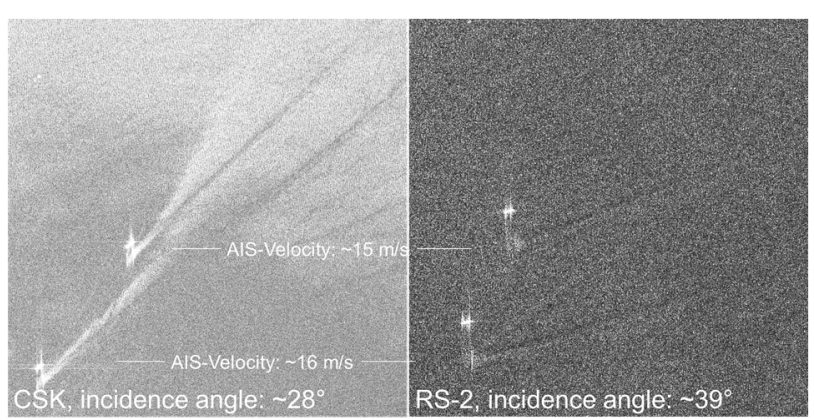

Figure 13 Comparison of CSK and RS-2 images acquired on 25.04.2019 at the same area with $\mathrm{HH}$ polarization and showing the same ships, left: CSK HIMAGE from $\sim 17: 11$ UTC, right: RS-2 Fine from $\sim 16: 43$ UTC

The figures show that the contrast of ship wakes with the ocean background is significantly more distinct on the CSK images compared to the RS2 images. However, in all three cases the incidence angle of the CSK image is lower than the incidence angle of the RS2 image. Thus, the comparison supports the characteristics of influences of the Incidence-Angle parameter as presented in subsection 2.1 .

\section{Discussion}

According to [5] all wake components except near-hull turbulences are better detectable under low incidence angles. For this reason, ship wakes consisting only of near-hull turbulences were excluded for this study. Consequently, the interdependent influence of the Incidence-Angle parameter on the detectability, which was postulated in [4], cannot be confirmed here. Instead, according to [5] the interdependent influence can now be explained by the fact that near-hull turbulences are better detectable under low incidence angles and in [4] all wake components were used in one model without separation.

The results in [5] are more recent than [4]. At the time when data was collected for the comparison conducted in this study, it was assumed that fast vessels would be better detectable under low incidence angles. This means, the originally planned qualitative comparison now lacks samples of RS2 images with lower incidence angle or CSK images with higher incidence angle in order to enable a qualitatively based comparison of wake detectability between RS2 and CSK.

Also, the study [3] is based on wake signatures without separation of wake components. It is possible that the variation in wake detection performance of TS-X, RS2 and S1 as presented in [3] is a result of better detectability of near-hull turbulences on TS-X than on RS2 and S1. In order to compare the wake detection performance between SAR sensors, besides the combination of sensors on similar orbit altitudes and radar frequency bands, also each wake component has to be considered separately.

\section{Conclusion}

In this study the X-Band SAR sensors TS-X and CSK and the C-Band SAR sensors S1 and RS2 have been utilized. In the former statistical analysis in [3] TS-X turned out to have better wake detection performance than S1 and RS2. In order to narrow down if the difference in orbit altitude or in radar frequency is responsible for the different performance, a comparison of CSK with RS2 images is conducted here. At the time when writing the paper, the problem cannot be solved. Only for S1 worse performance on the detection of ship wakes is observable in the results, while for RS2, TS-X and CSK similar performances are revealed.

\section{$6 \quad$ Literature}

[1] Graziano, M. D.: Renga, A.: Moccia, A.: Integration of Automatic Identification System (AIS) Data and Single-Channel Synthetic Aperture Radar (SAR) Images by SAR-based Ship Velocity Estimation for Maritime Situational Awareness. Remote Sensing. Vol. 11, No. 19, Sept. 2019

[2] Hennings, I.: Romeiser, R.: Alpers, W.: Viola, A.: Radar imaging of Kelvin arms of ship wakes. Int. J. Remote Sensing. Vol. 20, No. 13, 1999, pp. 2519-2543

[3] Tings, B.: Pleskachevsky, A.: Velotto, D.: Jacobsen, S.: Extension of Ship Wake Detectability Model for Non-Linear Influences of Parameters Using Satellite 
Based X-Band Synthetic Aperture Radar. Remote Sensing. Vol. 11, No. 5, Mar. 2019

[4] Tings, B.: Velotto, D.: Comparison of ship wake detectability on C-band and X-band SAR. Int. J. Remote Sensing.Vol. 39, No. 13, Jan. 2018, pp. 4451-4468

[5] Tings, B.: Non-Linear Modeling of Detectability of Ship Wake Components in dependency to Influencing Parameters using Spaceborne X-Band SAR. Remote Sensing. 2021 (submitted) 